\title{
Integrated course in psychiatry and literature during preclinical years and medical students' grades in the general psychiatry curriculum
}

\author{
Angela Pei-Chen Fan, ${ }^{1}$ Russell Oliver Kosik, ${ }^{1,2}$ Tung-Ping Su, ${ }^{1}$ Thomas C. Tsai, ${ }^{3,4}$ Wan-Jr Syu, \\ Chen-Huan Chen, ${ }^{1}$ Chen-Hsen Lee ${ }^{1}$
}

The Psychiatrist (2010), 34, 475-479, doi: 10.1192/pb.bp.109.028803

\begin{abstract}
National Yang-Ming University, Taipei, Taiwan; ${ }^{2}$ University of California, Los Angeles, USA; ${ }^{3}$ Stanford University School of Medicine, USA; ${ }^{4}$ Harvard School of Public Health, Boston, Massachusetts, USA
\end{abstract}

Correspondence to Angela Pei-Chen Fan (fan_angela@hotmail.com)

\begin{abstract}
Aims and method To examine the effect of taking an elective psychiatry and literature course during the first year of medical school on performance in the later mandatory general psychiatry curriculum. Class members were surveyed for baseline characteristics at the time of their admission to medical school. Following completion of their fourth year, average grades in psychiatry were calculated and results compared for those who did and those who did not take the course. Multiple regression analysis was used to assess the effects of baseline characteristics that were significantly different between the groups.
\end{abstract}

Results Students who took the course had statistically significant ( $t=-3.34$, $P<0.001)$ higher grades in fourth year psychiatry. They had lower admission interview scores $(t=-2.15, P<0.05)$ and reported less academic stress $(t=-9.55, P<0.01)$ before taking the course.

Clinical implications Literature is an effective medium through which to teach medical students psychiatry as it can lead to a greater understanding of the topic.

Declaration of interest None.
Teaching humanistic issues in medicine has become as important to medical schools as teaching more traditional courses such as pathology and physiology. ${ }^{1}$ It has been recognised that literature is an effective means by which to accomplish this. ${ }^{2-4}$ Teaching literature to medical students is a well-established tenet in numerous countries, particularly in the USA where over a third of medical schools are already doing so. ${ }^{1,5}$ Although once an innovative idea, the use of literature to teach humanistic issues in medicine is now the norm. Studies have shown that medical students who are taught via literature gain a deeper understanding of their patients and show more empathy towards them. ${ }^{6,7}$

As the use of literature has proven to be an effective means of getting across the complex subject of medical humanism to medical students, it follows that literature may be useful in teaching other aspects of medicine as well. Because of the importance of the patient-physician relationship and the role of communication in psychiatry, the teaching of psychiatric illnesses presents an opportunity to draw examples from literature. ${ }^{8}$ Psychiatric diseases are less tangible to students than physical illnesses, and this often leads to difficulties in students' capabilities to fully comprehend the mental disorders that they are studying. However, through literature they can see the person with a mental illness, the process of their treatment, the methods used by mental health professionals, and the inside of psychiatric hospitals. ${ }^{9}$ Students can also understand the entire set of circumstances in the life of a person with a mental illness, instead of only the illness or only the symptoms. ${ }^{10,11}$

Although many medical schools have employed literature to teach subjects such as medical humanism, very few have done so specifically to teach psychiatry. Among those that have, no one has shown in any quantifiable way whether the inception of such a course improves students' comprehension of psychiatry. We report here an innovative approach to teaching psychiatry using literature and the effect of such a course on students' performance in the fourth-year general psychiatry curriculum.

\section{Method}

The primary aim of this study was to measure the effect of completion of a course on psychiatry and Western literature in the first year of medical school on medical students' grades in the fourth year general psychiatry curriculum. The study sample consisted of all students in the class. At the time of entrance to medical school, these students completed a thorough baseline survey with questions related to their socioeconomic status, mental health and physical health (online Table DS1). We also collected data on 
students' academic performance including medical school grade point averages (GPAs), merits, demerits, medical school admissions interview scores, and scores on the national entrance examination (online Table DS1). Merit and demerit points are a supplementary evaluation system, provided by faculty for positive or negative student endeavours respectively.

Students had the option of taking the 'Psychiatry and Western Literature' course during their first year of medical school. Those who took the course comprised group 1 and those who did not take it, group 2. Following completion of the fourth year, we obtained each student's grade in the general psychiatry curriculum. This grade is determined by a midterm and final exam. The exams are based on lectures given by faculty of the psychiatry department and sporadic patient contact.

\section{Setting}

The National Yang-Ming University School of Medicine located in Taipei, Taiwan, was founded in 1971. Each year the school admits about 120 high-school students to begin the 7-year programme. Admission is determined by a variety of factors, the most important being the national placement exams administered by the Taiwan Ministry of Education. The vast majority of students are Taiwanese, although one or two places in each class are reserved for overseas students. In the first 2 years, students take general education courses and learn about the physician-patient relationship. During the following 2 years, classes focus on the basic sciences and clinical medicine. Students then progress to 2 years of required clinical rotations at the Taipei Veterans General Hospital and elective rotations at a range of other hospitals. The final year is the intern year, which in Taiwan is done before graduation from medical school. Students may do their intern year at the Taipei, Taichung or Kaohsiung Veterans General Hospital.

\section{Psychiatry and Western literature course}

Students taking this course meet for 2 hours once a week for 18 weeks. The course is offered in both autumn and spring semesters. It is an elective course available to first- and second-year medical students. Enrolment varies each year but averages about 40 students per semester.

The course has three goals. First, it aims to give students a general overview of the field of psychiatry and the most common psychiatric disorders. Second, it aspires to show the human features of these disorders through observation, personal reflection and group discussion of the literature. Finally, it attempts to introduce Taiwanese medical students to aspects of Western culture.

The course has one primary lecturer from the Department of Psychiatry, although at least one guest lecturer is invited to teach a session each semester. These guest lecturers have included artists, novelists, other psychiatrists and physicians, who focus their teaching on the integration of psychiatry and art as it applies to their field. For example, a session on operas with characters that have mental disorders is taught by a professional musician. The session considers the mentally ill character's arias and how they use melody to portray the varied emotions that the character experiences. Another session, taught by a
Table 1 Course materials and main psychiatric themes identified

Book Themes

Scarlet and Black, Socioeconomic status and self-esteem Stendhal Psychoanalysis

Sympathy, pride, the inferiority complex, ambition, the desire for revenge, jealousy, suspicion and regret

Crime and Punishment Social psychology and criminology and The Idiot, F. Dostoyevsky Depression Motivation for crime Good v. Evil, near-death experience, conscience, sacrifice, repentance and punishment

David Copperfield The impact of childhood trauma on and Oliver Twist, adolescent development, adult mental C. Dickens condition, and adult social judgement Erickson's, Piaget's and Freud's child and psychological development theories, including the Oedipus complex, role models, and the impact of the mother on the child are studied

Madame Bovary, Desire, satisfaction and fantasy G. Flaubert Addiction, obsessive-compulsive disorder, anxiety disorder and narcissism

\begin{tabular}{ll}
\hline Don Quixote, & Compromise between realism and \\
M. de Cervantes & idealism, or lack thereof
\end{tabular}

Delusion and paranoia

The Sorrows of Young Adolescent mental stress and disorders Werther, J. W. Goethe Adolescent depression, adolescent and The Catcher in the suicide, adolescent rebellion, hormone Rye, J. D. Salinger and development imbalance, and physical and mental development

The Metamorphosis, The patient's burden on the caregiver F. Kafka and their family

Stigma that accompanies some disorders and the loss of social identity that the person with a mental illness endures

\begin{tabular}{ll}
\hline Anna Karenina, & Depression \\
L. Tolstoy & Cognitive distortions \\
& Suicide \\
& Human psychological development \\
& Extramarital relationships \\
\hline
\end{tabular}

\begin{tabular}{ll}
\hline Jane Eyre, & Mentally ill spouse \\
C. Brontë & The rights of the person with a \\
& mental illness and the burden on \\
& the healthy spouse \\
& Schizophrenia and bipolar disorder \\
& Confinement of an individual with \\
& a mental illness who may be \\
& dangerous to others \\
& Social psychology, social oppression, \\
Ward No. 6, & and group pressures \\
A. Chekhov & Assimilation and conformity \\
and The Plague, & Mental disorder diagnosis \\
A. Camus & Hospital in-patient's well-being
\end{tabular}

novelist who has lived a large part of his life with depression, examines the trials of his own life and how those experiences became a part of his works. All lectures are given in Chinese with English interspersed.

\section{Course materials}

Each piece of course literature (Table 1) was chosen based on its relevance to a particular psychiatric topic. All works 
have Chinese translations. Students have the option of reading each book in English, although most prefer to read the Chinese versions. Students read either one or two books for each session.

The literature selection was designed to not only confront students with a variety of common psychiatric conditions but also to foray into the human consciousness and the spectrum of emotions that even a healthy individual will experience. It also addresses moral, philosophical and historical aspects of psychiatric illness and care.

\section{Data analysis}

We examined the differences between the group that took the course (group 1) and the group that did not (group 2). We calculated the mean general psychiatry grade for each group and performed a univariate analysis to look for a statistically significant difference. Further, we examined the baseline data for statistically significant differences between the two groups at the outset. We used regression analysis to assess the effect of those variables that we found to be both significantly different and liable to play a major role in the outcome. All analyses were performed with SPSS 17 for Windows.

\section{Results}

Group 1 had a higher percentage of students that were female, experienced less academic stress, and had a lower percentage of students who claimed to feel less healthy now than in the previous year, than group 2 (online Table DS1). However, of these, only academic stress was significant at $\alpha=0.05(t=-9.55, P<0.01)$.

Group 1 had higher national exam scores, lower interview scores, and more school demerits than group 2 (online Table DS1). Of these, only interview score was significant at $\alpha=0.05(t=-2.15, P<0.05)$.

Group 1 also had a higher mean cumulative GPA $(t=-1.49)$ and a higher mean first-year GPA $(t=-1.38)$ than group 2, although none of these results were significant (online Table DS1).

Students who took the course had higher grades in the subsequent fourth-year general psychiatry curriculum than those students who had not taken the course (mean 87.45 $($ s.d. =5.00) $\quad v$. mean $84.00 \quad$ (s.d.=5.80) respectively; $t=-3.34, P<0.01)$. These results were statistically significant both after performing univariate analysis and after performing multiple regression analysis (Table 2).

\begin{tabular}{|c|c|c|}
\hline & B & $95 \% \mathrm{Cl}$ \\
\hline Gender & $2.63^{*}$ & 0.50 to 4.76 \\
\hline Academic stress & 0.41 & -1.36 to 2.19 \\
\hline Feel less healthy than previous year & -0.25 & -1.58 to 1.08 \\
\hline Took Western literature course & $2.71 *$ & 0.51 to 4.92 \\
\hline
\end{tabular}

${ }^{\star} P<0.05$

$R^{2}=0.135$, ANOVA $=4.14, P<0.004$

\section{Student response}

After completion of the course, students recognised the difficulties of learning psychiatry in the traditional way and how the use of literature can combat these impediments:

'Psychiatric illnesses are problems which [a student] cannot fully understand. The teacher selected novels that gave a psychologically accurate depiction of the mentally ill, so that we could feel the spirit of the [psychiatric illness].

It is important that students were able to learn psychiatry in what one of them described as a more 'spiritual' way. Perhaps the sense of spiritual understanding came from the opportunity to view a psychiatric patient first-hand.

'Literature is a mirror of life. Through the readings this semester, we were lucky enough to spy on a number of different classical representations of human nature.'

The students used the literature to probe the human nature and the inner mind of an individual with a mental illness. Finally, the course piqued students' interest in psychiatry, an interest that they may not previously have had. One student wrote:

'When I first entered the university I did not have much interest in psychiatry ... but the literary works, even though I have not personally had the experiences [that the characters have had], have changed my own feelings towards psychiatry.'

Thus literature not only made psychiatry more accessible but also more appealing.

\section{Discussion}

The use of literature to teach humanistic issues in medicine has become commonplace in medical schools. ${ }^{5}$ We have shown that it can also be used to teach psychiatry. Why is literature such an effective medium through which to teach? First, literature tells stories. Throughout human history, stories have won our hearts and captivated our minds. Second, literature forces us to think in a way that we in the medical field may not be accustomed to. Literary analysis opens new doors, new worlds, worlds of metaphors and hyperboles, similes and symbolism. Educators should not underestimate the appeal of this kind of thinking in the medical student who spends their days memorising bacteria or calculating drug dosages. Finally, literature creates a personal connection between the reader and the characters. The reader experiences a character's illness in a vastly different way than reading about the illness in a textbook. ${ }^{10,11}$ Our students acknowledged this to be one of the fundamental reasons they gained so much from the course.

Although students in group 1 had two exposures to psychiatry, it is not likely that it was merely the extra exposure that was responsible for their improved scores in the general psychiatry curriculum. First, the material that the course covered was significantly different than the material that was tested on in the later psychiatry exam. Although the course did contain some amount of background information concerning major psychiatric disorders, the primary focus was to teach students the human side of these disorders through discussion of the literature, topics that were not tested on the exam. Second, the course was taken in the first year, but the psychiatry exam was not taken until the fourth year. The fact that students who took 
the course had scores that were significantly higher even after such a time lapse indicates that the improvements were probably not merely due to these students having extra lessons. Rather, the unique course design likely instilled in these students a more durable grasp of psychiatry as a field overall, one that will remain with them for a considerable amount of time.

\section{Limitations}

One limitation of the study was the non-random assignment of students to each group. Because the course was an elective, the decision whether or not to enrol may have led to differences between the two groups. To compensate for this, we identified those variables from the survey data that were significantly different between the two groups and used them in the regression analysis. Because there was no statistically significant difference in cumulative GPA and first-year GPA between the two groups, it is unlikely that one group was more intelligent or more motivated than the other. Those variables that were significantly different between the groups at $P<0.05$ included interview score and self-reported academic stress. Those variables that were not significantly different between the groups included gender, perception of current physical health as compared with the previous year, school demerits and national exam score.

It is unclear why students who took the course experienced less academic stress than those who did not take it. One would think that students who experience more academic stress would be more interested in taking such a course, so as to better understand their own mental health. On the other hand, perhaps it was academic stress that drove these students away from exploring a new course. Although some students view an innovative course design as a source of interest and excitement, those who become stressed over academic matters very easily sometimes view the same type of course as a potential source of uncertainty and anxiety. It is similarly unclear why students in group 2 were more likely to feel less physically healthy now than in the previous year than students in group 1. It seems that, at least in terms of academic stress and perception of physical health over the past year, students in group 2 were less healthy, both mentally and physically. Although students in this group were more likely to feel less physically healthy now than in the previous year, neither group was significantly more or less likely to feel less physically healthy than their peers.

Interestingly, students in group 1 had lower interview scores but higher national exam scores. Because all of the students were just beginning medical school at the time of course selection, their national exam scores served as the freshest measure of their academic abilities in comparison with their peers. Perhaps the success that students in group 1 experienced on their examinations gave them the confidence to try an atypical course, whereas students in group 2 were frightened away by a combination of their lower national exam scores and their higher amounts of academic stress. As interview scores remain confidential, even after admission, they could not play a role in influencing students' perception of their academic abilities in relation to their peers.

Literature seems to blend itself so well with other subjects that its integration with other aspects of the medical curriculum may be worth the consideration of educators. No one is suggesting that traditional courses, such as pharmacology, be eliminated but rather that literature could offer a new spin on them. For example, learning the side-effects of chemotherapy drugs from a textbook, although important, is not nearly as emotionally moving or memorable as reading the story of a person diagnosed with cancer and subsequently experiencing vomiting, anorexia and hair loss.

At the same time, medical curricula are cramped as they are and the addition of such a time-consuming subject as literature is one that absolutely must bear adequate fruit if it is to be included. Medical students in England were found to read fewer books that were unrelated to their studies once they started medical school. ${ }^{12}$ Even in our own course, one of the negatives that students mentioned was that the reading load was too heavy. Perhaps time considerations only allow certain subjects to be integrated with literature. If this is the case, then it is the job of educators to determine which subjects when integrated with literature will generate the greatest benefits to students.

In a 7-year medical programme, such as the one at National Yang-Ming University, curriculum designers have significant leeway to use integrated course designs. This may be more or less difficult to do in shorter or longer programmes.

Another consideration is whether these integrated courses should be made elective or required. Although most of the students surveyed at an English medical school thought that courses integrating literature and medicine were a good idea, the majority thought that these courses should be optional. ${ }^{12}$ They further believed that the integrated courses should not have exams. However, many courses that integrate literature with humanistic issues are required. ${ }^{7}$ This is clearly due to the principles taught in those classes (e.g. medical ethics, empathy) being deemed as universal traits of a good physician. But how in-depth should a medical student's knowledge of psychiatry be? Although it is important for every medical student to have some knowledge of psychiatry, not every student is cut out to be a psychiatrist. Further, some students are more attuned and stimulated by the subtleties of literature than others. Thus, in the same way that a clerkship student is required to do a general psychiatry rotation, although that same student has the choice to do an advanced psychiatry elective, medical educators must choose whether integrated courses should fall under the required or elective heading.

\section{Funding}

This study is supported by Ministry of Education of the Republic of China (Taiwan) grant MOE-096-03-03-2-11-3-02. The Ministry had no involvement in study design, data collection and analysis, interpretation of the results, writing of the report, and the decision to submit the paper for publication. The study was approved by the National Yang-Ming University institutional review board.

\section{About the authors}

Angela Pei-Chen Fan, Deputy Dean for International Affairs, Assistant Professor of Psychiatry, and Director for the Center for Faculty Development, Faculty of Medicine at National Yang-Ming University, Taiwan; Russell Oliver Kosik, MD/MPH student, University of California, Los Angeles School of Medicine, USA, and National Yang-Ming University 
International Health Program, Taiwan; Tung-Ping Su, Chairman, Department of Psychiatry, National Yang-Ming University, Taiwan; Thomas C. Tsai, MD/ MPH student, Stanford University School of Medicine and Harvard University School of Public Health, USA; Wan-Jr Syu, Dean for Academic Affairs, Taiwan; Chen-Huan Chen, Chairman, Faculty of Medicine, Taiwan; Chen-Hsen Lee, Dean, School of Medicine, National Yang-Ming University, Taiwan.

\section{References}

1 Hunter KM, Charon R, Coulehan JL. The study of literature in medical education. Acad Med 1995; 70: 787-94.

2 Jones $\mathrm{AH}$. Reflections, projections, and the future of literature-andmedicine. In Literature and Medicine: A Claim for a Discipline (eds D Wear, M Kohn, S Stocker): 29-40. Society for Health and Human Values, 1987.

3 McLellan MF, Jones AH. Why literature and medicine? Lancet 1996 348: 109-11.

4 Skelton JR, Macleod JA, Thomas CP. Teaching literature and medicine to medical students, part II: why literature and medicine? Lancet 2000; 356: 2001-3.
5 Charon R, Trautmann Banks J, Connelly JE, Hunsaker Hawkins A Montgomery Hunter K, Hudson Jones A, et al. Literature and medicine: contributions to clinical practice. Ann Intern Med 1995; 122: 599-606.

6 Macnaughton J. The humanities in medical education: context, outcomes and structures. J Med Ethics 2000; 26: 23-30.

7 Shapiro J, Rucker L. Can poetry make better doctors? Teaching the humanities and arts to medical students and residents at the University of California, Irvine, College of Medicine. Acad Med 2003; 78: 953-7.

8 Beveridge A. Should psychiatrists read fiction? Br J Psychiatry 2003; 182 385-7.

9 Bokey K, Walter G. Literature and psychiatry: the case for a close liaison. Australas Psychiatry 2002; 10: 393-9.

10 Oyebode F. Literature and psychiatry. Psychiatr Bull 2002; 26: 121-2.

11 Oyebode F. Literature and psychiatry. Adv Psychiatr Treat 2002; 8: 397-8.

12 Hodgson K, Thomson R. What do medical students read and why? A survey of medical students in Newcastle-upon-Tyne, England. Med Educ 2000; 34: 622-9.

\section{Prevalence of catatonic signs in acute psychiatric patients in Scotland}

The Psychiatrist (2010), 34, 479-484, doi: 10.1192/pb.bp.109.025908

${ }^{1}$ Royal Edinburgh Hospital;

${ }^{2}$ Whyteman's Brae Hospital, Kirkcaldy

Correspondence to Amal Al Sayegh

(amal.alsayegh@nhs.net)
Aims and method Concerns have been raised that catatonia is underdiagnosed. Prevalence varies (1.3-32\%) depending on diagnostic criteria. We used the Modified Rogers Scale to rate catatonic signs in patients consecutively admitted to three psychiatric wards over a 10-month period.

Results The prevalence of patients demonstrating any catatonic signs was at least 7.9-19.1\%. The most common catatonic signs were marked underactivity (not sedated), echolalia/palilalia, marked overactivity (not restlessness) and gegenhalten. In those with catatonic signs, the most common diagnoses were schizophrenia, schizoaffective disorder and dementia.

Clinical implications Most of the most common catatonic signs in our sample were motor signs. Antipsychotic-induced motor signs reflect interaction between drug and disease. Catatonic signs are not anchored in any one diagnosis and are on a spectrum of severity and quantity. Prevalence of these signs is higher than often presumed.

Declaration of interest None.
Kahlbaum characterised catatonia as a specific disturbance in motor functioning which represents a phase in a progressive illness that includes stages of mania, depression and psychosis that typically ends in dementia. ${ }^{1}$ Kraepelin incorporated features of catatonia into his concept of dementia praecox and Bleuler, under the heading of catatonic symptoms, gave the fullest account of schizophrenic abnormalities in movement, volition, overall behaviour and speech. ${ }^{2}$ Throughout most of the 20th century, clinicians considered catatonia as an exclusive subtype of schizophrenia. ${ }^{1}$ Numerous authors argued against this view as catatonic features amid affective symptomatology were not uncommon. ${ }^{3}$ Whereas until 1960 the catatonic subtype made up a third of the total number of cases of schizophrenia, the prevalence shrank to $2-10 \%$ during the following decades. ${ }^{4}$ The decline has been attributed to various causes, and includes early detection and management and a liberal and humane hospital atmosphere. $^{3}$ Other reasons include a more restrictive definition of schizophrenia; more reliable exclusion of other cerebral diseases by improved neurological diagnosis; improved capabilities of psychopharmacological treatment; 